\title{
The Effect of Personal and Relationship Dynamics as it Relates to Customs of Gift Exchanging and Vice Versa
}

\author{
Rea Karwal ${ }^{1}$
}

\begin{abstract}
Gift giving practices vary across cultures, occasions, and sentiments. The practice of giving and receiving gifts is often associated with displays of affection; and socially speaking, portray care, love, commitment and security. The purpose of this paper is to focus on personal as well as relationship dynamics that effect gifting rituals within romantic relations and vice versa. In order to do this, I shall be reviewing and discussing present literature central to the topics of love languages, attachment styles, gifting perceptions, commitment, and ulterior motives; all of which play critical roles in personal and relationship development.
\end{abstract}

Key Words: love language, attachment styles, perceptions, commitment, security, ulterior motives

\footnotetext{
${ }^{1}$ MacEwan University
} 


\section{Introduction}

Humans are social creatures and our nature posits that we all require social, emotional, physical, personal and familial connections in the form of various relationships, be they with parents, siblings, other family members, colleagues, friends, or romantic partners. Through the course of life, some relationships remain static, such as family relations while others can range from short, moderately or long lived due to life altering experiences, or as a result of natural decline, namely friendships and romantic relationships. Moreover, given our various needs as social creatures, expectations, behaviours, perceptions, and treatment, are critical in discerning whether certain companions are worth investing and maintaining relationships with for the purpose of securing our own wellbeing (Miczo, 2002).

Romantic relationships are especially pivotal in that the development and maintenance of this particular bond requires specific needs, desires and wants be met routinely (Hughes and Camden, 2020). As people mature, these requirements can alter with more current life stages, needs, goals and capabilities. Given the potential prospects associated with romantic relationships, aligned with consistent or evolving requirements, it is not uncommon for people to range from having a small quantity to an abundance of numerous partners throughout their lifetime, varying in length of time, satisfaction and levels of commitment. Generally, when seeking a romantic partner, one hopes that they will be long term; a life partner. Therefore, investment is necessary, especially for those with ambitions to marry, have children and/or pets, or pursue other grand plans which require special consideration, care, and attention. However, as many would surely attest, relationships are not so simple and straightforward. Many complex dynamics can interfere with, or affect relationship success as some social, emotional, physical, personal and familial needs can be detrimental to healthy functioning. However, some or all of these needs can possess personal limitations due to a culmination of shaping experiences, for example, poor emotional and relational development and regulation (i.e., attachment issues), insecurity, low self-esteem and confidence, poor boundaries and so on (Nguyen \& Munch, (2011). Comprehensive literature is dedicated to exploring the reasons behind these limitations and the resulting outcomes they incur on romantic relationships.

We can observe that, certain behaviours have been found to date back thousands of years, even across cultural and religious practices and differences (Hughes \& Camden, 2020, Bland \& McQueen (2018). Markedly, extensive literature has been devoted to researching and evaluating the concept of gifting practices in its varying capacities.

This literary review essay focuses on acts of gift giving, and how personal as well as relationship dynamics influence and affect this procedure, and vice versa. I hypothesize that given the inherent needs consistent with more successful relationships, gift giving is a practice that testifies to the consideration of these needs, due to the social and emotional component it has addressed in relationships across time and cultures. More specifically, topics that I will be addressing are as follows: gift giving as a love language, attachment styles and gift giving perceptions, signs of commitment and relationship security, ulterior motives of gift giving, and good and bad gifts. 


\section{Gift Giving as a Love Language}

Gary Chapman is famed for having written and published "The Five Love Languages" in 1992, and updating it in 2015 (Bland \& McQueen, (2018, p. 103). Met with unfathomable success, "The Five Love Languages" has been embraced by the likes of various helping professionals such as social workers, and psychologists. It has been employed internationally in government subsidized programs intent on improving relationships, by regular people globally (Bland \& McQueen, 2018). Chapman's five love languages consist of criteria specific to particular relationship needs: words of affirmation; such as compliments, kind words and requests, quality time; undivided, focused attention, reflective empathetic listening, and mutual enjoyment and participation of shared activities, acts of service; practical actions such as completing an everyday task for a partner, physical touch; tactile expressions of love ranging from handholding, kissing to sex, and lastly the focal love language for this essay, gifts; that are visual symbols of affection, which can be purchased, found or made (Bland \& McQueen, 2018; Hughes \& Camden, 2020).

When considering romantic relationship dynamics, love languages are an essential aspect to consider and implement with regards to emotional and physical bonding (Hughes \& Camden, 2020; Nguyen \& Munch, 2011). In their analysis, "Using Chapman's Five Love Languages Theory to Predict Love and Relationship Satisfaction", Jennifer L. Hughes and Abigail A. Camden explore Gary Chapman's love language theory in predicating love and satisfaction within romantic relationships (2020, p.234). They note that as per Chapman's theory, one of the main reasons for relationship problems is because couples speak different love languages (Hughes \& Camden, 2020). On average, people favour one or two love languages and have a natural propensity to show affection to their partners using their own preferred love languages (Hughes \& Camden, 2020; Egbert \& Polk, 2006; Bland \& McQueen, 2018). However, couples who report higher relationship satisfaction tend to express love with regard to their partner's desired love language(s) (Egbert \& Polk, 2006; Hughes \& Camden, 2020; Bland \& McQueen, 2018). Alternatively, those who do not express affection as it relates to their partners love language(s) can suffer from relational distress. Intriguingly, partners who display coinciding forms of love languages describe less distress within relationships overall (Bland \& McQueen, 2018).

Found as a fundamental expression of love across cultures, the exchange of gifts between partners exemplifies investment into the relationship; one that does not necessarily always require monetary value, but rather symbolic value (Hughes \& Camden, 2020; Bland \& McQueen, 2018). Most notably with regard to its situated practice; including objects, motivations, occasions and norms (Nguyen \& Munch, 2011).

Given these findings, gift giving practices are particularly meaningful when they showcase love and affection principally towards one's partner's specific love language. This has positive effects on the relationship as it demonstrates effort, communication and care; the reverse can be said for those who do not engage in their partner's love language of affections, and as a result, their relationship is more likely to be strained. 


\section{Commitment and Relationship Security}

Commitment and relationship security underlie the premise of romantic relationships. Without these elements, relationships possess little or no foundation upon which to stand and flourish. Literature has established research that extends and harmonizes with love language theory in exhibiting commitment and relationship security via their uses.

Actions affirming a partner's love languages can lead to higher reports of commitment and relationship security in that expectations pertaining to a partner's maintenance behaviours are being met (Edgard \& Polk, 2006). By contrast, failure to enact certain love language behaviours induces the opposite effect, potentially causing partners to feel unloved (Edgard \& Polk, 2006; Hughes \& Camden, 2020). The reasoning behind this is because learning to express a partner's love language often requires energy, effort and discipline. Furthermore, when done intentionally, it exhibits signs of love and commitment through the adaptation of their partner's love language into their own style (Hughes and Camden, 2020; Bland \& McQueen, 2018), especially when taking into account that intimate relationships often have a strong communal orientation towards mutuality rather than merely to the self (Luo et al., 2019). Relationship investments are defined as valuable resources; such as time, money, emotional and social energy and support, of which, should the relationship be terminated, both partners would lose their investments. Therefore, it can be generalized that investments made by each individual partner acts as a catalyst of motivation for commitment towards each other (Christian, Sellbom \& Wilkinson, 2017).

Not only does conveying a partner's love languages support love and commitment, it also opens channels for deeper communication, along with expansion with regards to self-growth, experience, and realizing underdeveloped qualities that can be integrated into individual personalities. When involved with a romantic partner, one's sense of self and identity is not diminished when providing a partners desired love language; but rather both partners connect in a deep and meaningful way such as; working together as a team, encouraging and supporting each other, and helping each other achieve meaningful goals (Bland \& McQueen, 2018).

\section{Attachment Styles, Perception and Gift Giving}

John Bowlby, a British psychologist, psychiatrist and psychoanalyst (Bretherton, p. 759760), is highly regarded for his pioneering work pertaining to child development and attachment theory. In several publications along with Mary D.S. Ainsworth, who examined individual differences in attachment, Bowlby revolutionized future understandings of perceptions and behaviour rooted in adverse child events, now more commonly referred to as ACE's (Crittenden, 2017; Bretherton, 1992). Bowlby reconceptualised Sigmund Freud's perspective on children as clingy and needy, and counter-argued that rather, they are competent, curious and fully engaged with the environment (Waters, Crowell, Elliot, Corcoran, \& Treboux, 2002). Moreover, Bowlby declared that in order for a child to mature in a mentally healthy way, infants and children must grow up involved in warm, intimate and continuous relationships with their mother or substitute parental figure (Bretherton, 1992). Bowlby's study of the role of early experiences on infant- 
mother and then adult-adult relationships reflect different forms of attachment: secure, insecure, and avoidant. Bowlby also developed control systems theory, which accounts for intricate monitoring of internal states: relationship experience, proximity seeking, communication from a distance, and distancing from attachment figures (Waters, Crowell, Elliot, Corcoran, \& Treboux, 2002). For this reason, it can be inferred that attachment styles may affect gifting perceptions and behaviours as a result of healthy or unhealthy associations of social interactions and customs during punitive years of relational and social training and learning. As such, Bowlby is accredited in an abundance of literature in connection with child and adult perceptions, behaviours and forms of attachment and relationships.

Attachment theory draws heavily on the role of relational experiences in shaping behaviour. In particular, this theory suggests that it is through the development of bonding and proximal efforts that people form "internal working models" of the self and others with regards to varying relationships. This progress begins during infancy, a time when there is an intrinsic need to bond as well as conserve proximity to others, namely parents, as a means of avoiding potential threats, and ensuring security (Christian, Sellbom \& Wilkinson, 2017, p. 330). Moreover, attachment styles regulate inner distress; including but not limited to anxiety, anger, depression and emotional detachment (Nguyen \& Munch, 2011). As infants mature into adulthood and beyond, their internal working models of cognition and behaviour generalise to other relationships: friendships, romantic relationships and so forth (Christian, Sellbom \& Wilkinson, 2017). Therefore, attachment styles play a crucial role in personal as well as social development; especially as it pertains to romantic relationships.

When evaluating gift giving as a love language and its effect on commitment, consideration must be given for attachment styles and how one may perceive and receive or decline affection (Nguyen \& Munch, 2011; Nguyen \& Munch, 2011). In Bland and McQueen's study, “Five Love Languages Cluster Analysis", they note that Chapman himself alludes to the role formative experiences play in influencing a person's primary love languages (2018, p. 121). To clarify, love languages are developed within the context of the progression of different forms of attachment and love, starting at birth with parents; this matures into passionate love with romantic partners and based on unresolved attachment issues, love languages are used to compensate for needs unfulfilled by one's parents. Set in childhood, love languages tend to remain consistent throughout the lifespan. Within their study, Bland and McQueen (2018) address some significant findings relating to gift giving and attachment styles. First, receiving gifts was generally preferred by women. Second, receiving gifts was valued greater by women than physical touch (which may mirror their preference for material objects over human contact as per avoidant attachment dynamics during times of distress). Lastly, East Asian couples had a higher tendency to use gift giving as a form of affection likely because of cultural norms within the cluster of relationship congruence through all stages of a relationship (Bland and McQueen, 2018; Cong et al., 2018).

Individual differences in poor attachment styles can generally be classified into two alternate dimensions: attachment anxiety (evolving from a history of inconsistent responses to attachment attempts) and attachment avoidance (evolving from a history of insensitive and nonresponsive caregiving). (Christian, Sellbom, Wilkinson, 2017; Nguyen \& Munch, 2011). Behaviours and preoccupation indicative of attachment anxiety include a fear of abandonment, doubts of self-worth, and excessive reassurance seeking of the availability of others (Christian, 
Sellbom, Wilkinson, 2017). Feeling unworthy of love and seeing others as unreliable or untrustworthy were also identified by Nguyen \& Munch, 2011). Conversely, behaviours and preoccupations indicative of attachment avoidance include an avoidance of emotions, dependency, intimacy, defensive self-inflation, and cynicism regarding relationships (Christian, Sellbom, Wilkinson, 2017), being emotionally aloof, independent, distrusting of romantic partners and maintaining autonomy and emotional distance (Nguyen \& Munch, 2011). Secure attachments however, are promoted through healthy, responsive and consistent caregiving, and as such, is characterized by positive self-perception, feelings and acceptance of worthiness and love, viewing others as trustworthy and reliable, and confidence in seeking comfort and support (Nguyen \& Munch, 2011; Waters, Crowell, Elliot, Corcoran, \& Treboux, 2002).

Specific gifting paradigms at this time are also important to clarify, given that attachment styles can affect gift giving, receiving practices and perceptions (Nguyen \& Munch, 2011). As mentioned in their study, "The Moderating Role of Gift Recipients' Attachment Orientations on Givers' Gift-giving Perceptions", the researchers outline gift giving in three contextual exchanges: 1) economic exchange; 2) social exchange; and 3) agape love. In the economic exchange paradigm, gift giving is used as a means of initiating a relationship and gaining proximity to a romantic partner, such as coffee dates. In the social exchange paradigm, gifts are given and perceived as symbols of commitment, such as Valentine's day or birthday gifts. Lastly, in the agape love paradigm, gift giving is selfless, expressive, and does not require reciprocity. Within relationships, economic and social exchanges prevail early, yet as the relationship evolves into deeper, more and committed stages, agape love begins to predominate.

Delving further into attachment styles as they pertain to perceptions of gift giving, receiving and reciprocity, Nguyen and Munch (2011) reported that for some, gift exchange practices are deemed as an enjoyable, noble expression of love and commitment, whereas others view it as a social obligation and chore. For the latter, Nguyen and Munch concur that "the obligation to give may be based on moral or religious imperatives, the need to recognize and maintain a status hierarchy, the need to establish or maintain peaceful relations, or simply the expectation of reciprocal giving" (p. 114). In their study, "Romantic Gift Giving as Chore or Pleasure: The effects of Attachment Orientations on Gift Giving Perceptions", Nguyen and Munch hypothesised, examined and found three different attachment styles and their corresponding gifting behaviours (2011, p. 114). They explain that due to fears of abandonment or neglect, individuals with anxious attachments tend to blame partners for being unsupportive and not meeting their emotional needs. A strong focus is placed on their partners and they constantly assess whether they are providing them with enough attention. Due to insecurity and fears, high value is placed on securing their relationship, especially via the use of gifts. Finally, those with avoidant attachment styles invest less than others in romantic relationships due to low expectations of support, they value autonomy, experience low levels of intimacy and are therefore unwilling to change.

For these reasons, it is expected that these individuals perceive gift giving more as an obligation due to fear and concerns that first, gifts will be misinterpreted due to their emotional nature (Nguyen \& Munch, 2011; Luo, Fang, Shen \& Cong, 2019) or, in other words, perceived as disingenuous due to lack of congruence with normal cognitions and behaviours they present. Secondly, because gifts are a sign of commitment which they fear (Nguyen \& Munch, 2011). For secure attachments, Nguyen and Munch (2011) propose that as it is low on both anxious and 
avoidant thoughts and behaviours, having a secure sense of self and love (i.e. those who are securely attached) have no fear of intimacy with romantic partners. Therefore, they make considerable investments into relationships, perceiving gift giving as an ideal means of expressing love and not as an obligation. Interestingly, Nguyen and Munch also discuss self-esteem as it correlates with attachment theories. More specifically, they assert that given dysfunctional attitudes associated with insecure attachment styles, this predisposes people to lower levels of selfesteem (Nguyen \& Munch, 2014; Hyun, Park \& Park, 2016), which may influence a "more is better" philosophy when assessing proximity and reassurance from partners. Consequently however, this may promote more evidential security seeking as opposed to actual security and healthy relationship functioning (Nguyen \& Munch, 2014).

\section{Gift Giving and Ulterior Motives}

Not all gifts are intended to further or fully maintain relationships. People with hidden agendas may use gifts as a way to manipulate, guilt, or self-gratify themselves against unsuspecting or dependent partners. In this, they employ power plays to maintain control over their victims, as well as their self-image depictions to themselves, or the people surrounding them. This evaluation not only stems from literature, but also from personal experiences of which I will elaborate at the end of the following literary review.

Self-congruence theory stipulates that individuals conduct themselves in ways that align with their own self-image (Cong et al., 2018). Furthermore, making choices that conform to one's self-image encourages self-positive emotions. This is especially true when relating to gift giving and the opportunity to portray oneself with regard to image and presentation (Cong et al., 2018; Hyun, Park \& Park, 2016). Research intended to evaluate the importance of self-image on gift giving etiquette explored individual differences, significantly with regard to global attachment (as previously discussed), personal values, cultural norms and most strikingly, self-esteem and narcissism (Cong et al., 2018). In their analysis set out in "Narcissism and Gift giving: "Not every Gift is for Others", Hyun, Park and Park (2016) investigate the relation of self-esteem and narcissism on gift giving correspondence. Self-esteem refers to "feelings of adequacy" (p. 48). Those with high levels of self-esteem in romantic relationships are likely to embrace a passionate love style, have positive views of their partner and hence, and not view gift giving or reciprocity as an obligation. It can be assumed then that the reverse can be said for those with low self-esteem.

Further, with respect to narcissism, it has been correlated with low levels of commitment, satisfaction and investment. Additionally, narcissistic individuals' relationships are sought as a means to obtain admiration, power and sexual resources. In Hyan, Park and Park's study, 105 Korean, heterosexual, unmarried couples completed a measure of narcissism and self-esteem and gift motivation scale, covering past experiences and behaviour as well as a future hypothetical scale if monetary concerns were no issue. Self-esteem was measured through the Rosenberg selfesteem inventory, and narcissism was measured using a 40-item questionnaire narcissistic personality inventory rated on a 7-point scale. Gift giving motivation questions were constructed based on past literature and collaboration with 15 undergraduates majoring in psychology and consumer behaviour who posited personal and general trends in romantic relationships. In their 
findings, they reported that self-esteem negatively correlated with power motivation, and those who reported high self-esteem would not engage in gift giving as a maintenance motivation in the future. Contrarily, a positive correlation was found between narcissism, power motivation and maintenance motivation. This particular finding has been found consistent across cultures as a means of encouraging the self-enhancement bias (Hyun, Park \& Park, 2016).

Although they do not directly address gift giving practices, Elliot Christian, Martin Sellbom and Ross B. Wilkinson in their study, "Clarifying the Associations Between Individual Differences in General Attachment Styles and Psychopathy" (2017), compare and contrast normal modes of behaviour and psychopathy in relation to general attachment styles. They first claim that psychopathy is a construct widely associated with antisocial interpersonal behaviours defined by characteristics of callousness, lack of empathy, egocentricity, manipulation, fearlessness, irresponsibility, and impulsivity. As it is defined by interpersonal problems (i.e., lack of empathy, manipulative and poor bonding), it can be assumed that those diagnosed with psychopathy seek to fulfill self-cognitive schemas, dominance and hostility. Psychopathy shares commonalities with insecure avoidant attachment style and causal qualities such as poor parenting, abuse, maternal depression and low economic status. However, given its varying and inconsistent nature, it is likely the outcome of disorganized attachment. Through a 249 subject analysis of self-reports of psychopathy consisting of a triarchic measures of psychopathic traits (i.e. boldness, meanness, disinhibition, egocentricity, callousness, antisocial, attachment avoidance, and attachment anxiety inventory measures, as well as a 40 item attachment style questionnaire, Christian, Sellbom and Wilkinson summarized that more attention is needed in addressing this topic as results were inconsistent with anxious and avoidant attachment types. Moreover, certain characteristics could be attributed to other means, for example, boldness could correlate with self-confidence.

Despite the lack of review in psychopathy and perspectives of gift giving, but given the recognition of this construct as an antisocial personality disorder (as with narcissism in the prior literary review), it can be strongly hypothesized that those diagnosed with psychopathy may recreate similar trends. Especially with regard to tactics of manipulation, control and hostility as well as maintaining a positive self-image to themselves and others; it can be seen as a motive for gifting practices rather than as a means to display affection or commitment.

\section{Personal Experience with Ulterior Motives}

Continuing to my personal experiences in a toxic relationship, I can attest to the qualities congruent with narcissism and psychopathology in esteem to gifting motives, as well as personality traits. My ex-boyfriend had once admitted to me that his therapist had told him he almost met the requirements of being diagnosed a psychopath. Through his use of manipulation beginning at the start of our friendship, to the end of our romantic relationship; namely in the form of grand lies inferring he was increasingly depressed, had poor personal connections, avoided conflict, had alcoholic and neglectful parents, was cheated on, went into foster care, and so on, he garnered my sympathy, and affection. Given that I myself am an anxiously attached person, I grew strongly attached quickly, and would consistently require self-assurance in the forms of words of affection and quality time. Being that he sought to use me for his own emotional, social, and monetary 
needs, he would mirror my personality, wants, needs and desires so that I became accustomed to hostile and loving cyclic behaviours. Even though I realized his stories did not add up, his behaviour did not reflect actual care or commitment; and when I would try to discuss these matters, I was made to feel guilty for reflecting on the past, that my own issues were causal of our relational dysfunction, or that because he had been through so much he couldn't help his negative qualities. I generally tend to people please heavily, due to my own issues with abandonment, to secure my relationships. This was no exception with him, he abused this quality of mine. When it came to acts of gift giving, I would buy multiple personalized items reflecting his personality and likes, spend large amounts of time and money to do so, for all occasions: birthday, Christmas, and Valentine's Day. He, however, never bought gifts for me for these occasions. He made promises that he had ordered an item online as a Christmas or birthday present, but there were issues with delivery, or his banking account, or if we had a fight and I broke up with him, he would "cancel" the order. In the entire time we dated, I never received that gift. For nights out, I was often the one paying for dates, he would pay sometimes if the bill was cheap enough, or if I had complained he wasn't making an equal effort, just to maintain control and prove he was trying to better himself within the relationship for my sake.

\section{Good and Bad Gifts}

Personal preferences may dictate what constitutes a good or bad gift; whether it does or does not coordinate with our wants, needs, personality, or stylistic choices. The difference between good and bad gifts also relies on situational contexts. For example, some gifts correspond with social and cultural norms and are appropriate for certain occasions such as flowers, chocolates or jewelry for Valentine's day, expensive electronics, perfumes, clothes for birthdays and Christmas.

In their study, "The Gift of Similarity: "How Good and Bad Gifts Influence Relationships", Elizabeth W. Dunn, Jeff Huntsinger, Janetta Lun and Stacey Sinclair (2008) assess good and bad gifts as markers of interpersonal similarity. They first begin by observing that gift giving is central to many occasions such as birthdays, graduation, Christmas, and so on. They then discuss how literature acknowledges that similar gift giving styles serve as a marker for compatibility in terms of similar interests and tastes. Furthermore, they note that those who share similarities with their partners were more accurate in predicting their partners' preferences. As an aside, Dunne et al., also refer to other literature that corroborates the fact that poor gifting choices can have negative impacts on relationship satisfaction overall. In order to analyze their hypothesis, Dunn et al., conducted two similar experiments. In the first, new opposite sex acquaintances would choose desirable or undesirable gifts. The second was identical with the exception of substituting current romantic partners with those of new acquaintances. The procedure for this experiment was as follows: Each participant was asked to rank-order their desire to win a $\$ 20$ gift certificate (for participation and completion of the study), and then choose a desired location based on eleven different companies. They would then be able to select a lottery ticket for their partner and vice versa. The participants then received their lottery ticket from their corresponding partners (romantic and acquaintances). Unknown to them however, the experimenter entered in contrasting 
answers and in place of correct ones, would enter the participants' least preferred options, randomly assigning each subject pair to bad or good variables. Subjects were then asked to communicate their views of similarities to their partner on a scale of 1-8 (Dunn et al., p. 472). For experiment one, the hypothesis was confirmed in that men felt less similar to women after receiving a bad gift. For experiment two, men perceived less similarity to their romantic partners after receiving a bad gift, whereas women reported a significantly greater rate after receiving a bad gift. As a final overview of their findings, Dunn et al. concluded that men in both situations (acquaintance and romantic partner) felt lower levels of similarity with their female counterparts. Contra wise to this, women tended to overcompensate for their partners poor gift choice by claiming higher reports of similarity.

This specific study is important as little research has been done on gift giving and perspectives of similarity when considering alternative forms of relationships and levels of possible potential and drawbacks (particularly, with the perceptions between good and bad gifts). The results allude to certain gender differences that are compatible with other studies where it was found that men and women retain alternating mindsets with regard to gift giving. (See Bland \& McQueen (2018), Luo et al. (2019), and Cong et al. (2018)).

\section{Discussion}

In summation, congruent with my hypothesis based on an analysis of the literature that is available, customs of gift giving do have an effect on romantic relationships with regard to being dependant on love languages, signs of commitment and satisfaction, attachment styles, perceptions of gift giving, ulterior motives and good versus bad gifts, and vice versa. Much literature was found to overlap in agreement of how these factors individually and collectively influence and effect relationships in positive and negative ways. Particularly enlightening were discussions regarding Chapman's five love languages, Bowlby's attachment theory and displays of commitment. The act of affirming a partner's love language, in this case gifts, is vital in that it exemplifies care, effort, and commitment on their part. Furthermore, gift giving in itself is seen as an act of commitment, due to the amount of personal, emotional, and monetary investment gifting portrays. These facts coincide with relationship satisfaction overall. Attachment styles indicate differential perceptions and behaviours concerning gift giving and receiving, especially as they are situated in anxious, avoidant and secure personal and relational dynamics. Ulterior motives alluded to personal, self-serving incentives benefiting those with narcissism or narcissistic traits with little to no actual consideration of their partners. Lastly, good versus bad gift giving was associated with poorer perspectives on similarity for men when receiving bad gifts, whereas women scored higher rates of similarity in this same instance, however they are more inclined to make more efforts in sustaining relationships.

As a final comment, more research needs to be conducted regarding ulterior motives of gift giving, with respects to personality disorders. This includes more in-depth studies into gifting practices of those with diagnoses of narcissism and psychopathy, and for those with other personality disorders (e.g. sociopathy, borderline personality disorder, paranoid personality disorder and so on). Moreover, further research is needed on opinions regarding what constitutes "good" versus "bad" gifts for both males and females. 


\section{References}

Bland, A. M., \& Mcqueen, K. S. (2018). The distribution of Chapman's love languages in couples: An exploratory cluster analysis. Couple and Family Psychology: Research and Practice, 7(2), 103-126. https://doi.org/10.1037/cfp0000102

Bretherton, I. (1992). The Origins of Attachment Theory: John Bowlby and Mary Ainsworth. Developmental Psychology, 28(5), 759-775. https://doi.org/http://dx.doi.org/10.1037/00121649.28.5.759 10.1037/0012-1649.28.5.759

Christian, E., Sellbom, M., \& Wilkinson, R. B. (2017). Clarifying the Associations Between Individual Differences in General Attachment Styles and Psychopathy. Personality Disorders: Theory, Research, and Treatment, 8(4), 329-339. https://doi.org/http://dx.doi.org/10.1037/per0000206

Cong, R., Luo, B., Li, T., \& Wang, C. (2018). It is for you, or it is for me: How relationship dependence affects gift image consistency in romantic relationships. Journal of Consumer Behaviour, 17(4), 343-354. https://doi.org/ https://doi.org/10.1002/cb.1724

Crittenden, P. M. (2017). Gifts from Mary Ainsworth and John Bowlby. Clinical Child Psychology and Psychiatry, 23(3), 436-442. https://doi.org/https://doi.org/10.1177/1359104517716214

Dunn, E. W., Huntsinger, J., Lun, J., \& Sinclair, S. (2008). The Gift of Similarity: How Good and Bad Gifts Influence Relationships. Social Cognition, 26(4), 469-481. https://doi.org/10.1521/soco.2008.26.4.469

Egbert, N., \& Polk, D. (2006). Speaking the Language of Relational Maintenance: A Validity Test of Chapman's (1992) Five Love Languages. Communication Research Reports, 23(1), $19-26$.

Hughes, J. L., \& Camden, A. A. (2020). Using Chapman's Five Love Languages Theory to Predict Love and Relationship Satisfaction. Psi Chi Journal of Psychological Research, 25(Special Issue 2020), 234-244. https://doi.org/10.24839/2325-7342.JN25.3.234

Hyun, N. K., Park, Y., \& Park, S. W. (2016). Narcissism and gift giving: Not every gift is for others. Personality and Individual Differences, 96, 47-51. https://doi.org/10.1016/j.paid.2016.02.057

Luo, B., Fang, W., Shen, J., \& Cong, X. F. (2019). Gift-image congruence and gift appreciation in romantic relationships: The roles of intimacy and relationship dependence. Journal of Business Research, 103, 142-152. https://doi.org/https://doi.org/10.1016/j.jbusres.2019.06.018

Miczo, N. (2002). Hobbes, Rousseau, and the "Gift" in Interpersonal Relationships. Human Studies, 25(2), 207-231. doi:10.1023/a:1015588007217

Nguyen, H. P., \& Munch, J. M. (2011). Romantic gift giving as chore or pleasure: The effects of attachment orientations on gift giving perceptions. Journal of Business Research, 64(2), 113-118. https://doi.org/https://doi.org/10.1016/j.jbusres.2010.02.006

Nguyen, H. P., \& Munch, J. M. (2014). The moderating role of gift recipients' attachment orientations on givers' gift-giving perceptions. Journal of Consumer Behaviour, 13(5), 373-382. https://doi.org/10.1002/cb.1486

Waters, E., Crowell, J., Elliot, M., Corcoran, D., \& Treboux, D. (2002). Bowlby's secure base theory and the social/personality psychology of attachment styles: Work(s) in progress. Attachment and Human Development, 4(2), 230-242. https://doi.org/10.1080/14616730210154216 